\title{
АКТУАЛЬНОСТЬ ПРИМЕНЕНИЯ ТЕОРИИ НЕЧЕТКИХ МНОЖЕСТВ ПРИ ПЛАНИРОВАНИИ РАЗРАБОТКИ МЕСТОРОЖДЕНИЙ ПРИРОДНОГО ГАЗА С УЧЕТОМ НЕОПРЕДЕЛЕННОСТИ ИСХОДНЫХ ДАННЫХ
}

\section{THE RELEVANCE OF USING THE THEORY OF FUZZY SETS IN PLANNING THE DEVELOPMENT OF NATURAL GAS FIELDS, TAKING INTO ACCOUNT THE UNCERTAINTY OF THE INITIAL DATA \\ A. Chuvashov P. Kozhevnikova}

Summary. Improvement of methods for calculating hydrocarbon reserves and expedient methods for the development of natural gas fields is a rather urgent issue at the current stage of development of the oil and gas industry. The need to modernize these methods is dictated by the task of quickly entering the development of hydrocarbon deposits on the basis of an accurate assessment of hydrocarbon reserves in the shortest possible time and with low investment of funds. In a market economy, the production plan cannot be considered strictly specified, therefore, optimal plans are sought that maximize such a criterion as the cumulative production of a group of fields; the approximation-combinatorial calculation method is used in combination with the branch-and-bound method. In addition to the optimal one, methods that are close to it are sought, since this allows performing multicriteria optimization for nonmain assessment markers. At this time, work is underway to improve the complex to take into account the fuzziness of the initial data, based on the use of the apparatus of fuzzy sets. And, although there are clear advances in various parts of the problem of developing hydrocarbon deposits in conditions of fuzzy, uncertainty and fuzzy block of information of the initial data, in general the state of its solution is not ideal. For the most part, the reason is that the studies are rather fragmentary and also have little coverage of the problem. Based on this, fundamentally new methods of creating a design complex for the development of oil and gas fields in the aforementioned conditions and current geological and field information are needed. A comprehensive solution in this case is one of the scientific directions for increasing the value of the coefficient of hydrocarbon recovery from reservoir rocks. One of its key advantages is the fact that oil and gas deposits are considered as a whole and, at the same time, their individual components are taken into account. The use of functions of fuzzy sets in the description of objects of development and assessment of methods of influence does not exclude an integrated approach, but, on the contrary, constitutes its initial natural basis.

Keywords: natural gas reserves, fuzzy set, development of gas fields, hydrocarbon deposits, indicators of development of gas fields.

\author{
Чувашов Артур Александрович \\ Аспирант, Ухтинский государственный \\ технический университет, г. Ухта \\ sid_artur@mail.ru \\ Кожевникова Полина Валерьевна \\ К.т.н., дочент, Ухтинский государственный \\ технический университет, г. Ухта \\ pkozhevnikova@ugtu.net
}

Аннотация. Усовершенствование способов подсчета запасов углеводородов и целесообразных методов разработки месторождений природных газов является достаточно актуальным вопросом на текущем этапе развития нефтегазодобывающей промышленности. Необходимость модернизации данных методов диктуется задачей быстрого ввода в разработку месторождений углеводородов на базе точной оценки запасов углеводородов в кратчайшие сроки и при невысоких вложениях денежных средств. При таких представленных подходов, когда превалирующую роль приобретают процессы коммерческого уклада в экономической составляющей, не поддается точной оценке планы с точки зрения их оптимистичности. Если методология выбрана оптимально, то соответственно потребуется дополнительная оптимизация, которая строится на комплексе факторов с учетом массива маркеров. В настоящее время еще актуально проведение работ по гармонизации комплексных мер, которые будут являться органичной структурой стартовых данных, что базируется на методологии нечеткого массива множеств. И, хотя есть явные достижения по вариативным частям проблемы извлечения углеводородов из месторождений в условиях нечеткости, неопределенности и размытости комплекса информации исходных материалов, в общем прогресс ее решения не является идеальным. По большей части причина заключается в том, что исследования носят довольно фрагментарный характер, а также имеют небольшой охват данной проблемы. Исходя из этого, необходимы принципиально новые методы создания комплекса проектирования разработки месторождений нефти и газа в выше обозначенных условиях и текущих геолого-промысловых данных. Комплексное решение данной проблемы — одно из направлений увеличения числа коэффициента извлечения ископаемых из пород-коллекторов. Одним из его основных достоинств является то, что залежи углеводородов рассматриваются как единое целое и, параллельно с этим, ведется учет и их отдельных составляющих. Применение функций неопределенных множеств при представлении объектов разработки, а также оценки способов влияния не исключает совокупного подхода, а, напротив, составляет его начальную естественную базу.

Ключевые слова: запасы природного газа, нечеткое множество, разработка газовых месторождений, залежь углеводородов, показатели разработки газовых месторождений. 


\section{Ввемение}

«П лан по развитию эффективного способа добычи газа», имеющий в основе имитационную модель комплекса месторождений для проектирования их разработки в Сибири, активно применялся в конце XX века. Другим его типом является «План моделирования и усовершенствования процесса добычи газа». Современная экономика становится все более нацеленной на коммерцию, ввиду чего план извлечения имеет размытые границы, а это, в свою очередь, обуславливает появление других более-менее приемлемых планов, которые увеличивают критерий аккумулированной добычи комплекса месторождений в наибольшей степени; для полной эффективности пользуются комбинаторно-аппроксимационным методом расчета, варьируя его с другим методом - ветвей и границ. Разрабатываются также и другие методы, по своему функционированию приближенные к данному, т.к. это дает возможность осуществить оптимизацию по всем уровням, даже по дополнительным пунктам, с помощью которых проводится оценка. Специалисты прибегают к новым методам исследований, потому они проводят специальные работы, благодаря которым улучшение комплекса для учета неточности исходных данных проходит на должном уровне, потому в процессе его функционирования специалисты руководствуются комплексом неточных множеств [1].

Осветим чуть более подробно аспект неточности исходных данных. Результаты практической деятельности не раз демонстрировали, что постановки и методы решения определенных задач из всех методов более популярны, однако зачастую в прикладных задачах мы можем иметь дело с такой характеристикой, как неточHOCTь.

Когда на практике используют случайный тип неточности, то существует мнение, что неизвестные компоненты, которые стабильны по статистическому маркеру, выступают в качестве случайных величин с исходными законами разверстки. В ситуациях неточности случайного типа мы не имеем никакого права применять базовую статистическую выборку, т.к. количество наблюдений, необходимых для проверки осуществления любого распределительного закона, недостаточно, или же объекты, выбранные для рассмотрения, являются по своей структуре совершенно разнообразными [2].

Не только имитационные, но и те модели, что предназначены для нахождения лучшего решения при соблюдении заранее определенных условий и ограничений, системы [3] указывают на свою полную достоверность, что, следует сказать, не до конца верно; к примеру, не- сколько характеристик месторождений гораздо уместнее считать адетерминированными: дебиты различных скважин, стоимость газа, различные категории запасов газа, уровень так называемой «полки» (стабильного значения добычи углеводородов), и т.д.

Обоснование возможности применения комплекса нечетких множеств. В большинстве случаев, с целью определения неопределенностей в добывающем деле, используется комплекс стохастического программирования, а также теории вероятностей, но не так давно появились научные труды, главной целью которых было осветить то, насколько оптимально применяются в практической деятельности нечеткие множества $[2,4]$.

Совокупность нечетких множеств обладает авторитетными достоинствами (если сравнивать ее с другими способами учета неточностей:

1. рациональнее всего использовать базовые вероятности для одинаковых случайных событий, для которых применимо определение случайных настроек с заданными распределительными функциями и их вспомогательными компонентами [5]. Однако компоненты неточности с точки зрения экономики и хозяйства в целом не позиционируют себя в качестве статической базы, причем, даже сами объекты зачастую являются исключительными;

2. в ходе рабочего процесса, в котором превалируют нечеткие множества, взаимоисключается большинство различных проблем, которые возникают из-за использования недостаточно достоверных исходных условий:

- сущность неточности проявляется конкретно посредством нечеткости;

- не нужно тщательно описывать вид и настройки плотностей распределения для комплекса недостаточно достоверных исходных условий (в силу того, что зачастую это просто невозможно выполнить);

- в поле зрения специалиста находятся фактические данные о рассеивании численных значений настроек, а не информация, касающаяся неявных оценок (в том числе и вероятностных показателей);

- значения величин с разными типами неточности (предположим, данные, значения которых записаны в равноотстоящих друг от друга точках в определенной шкале) выражены более конкретно, благодаря концептуальному языку нечетких множеств;

- возможность использования в коммуникационной деятельности различные вербальные понятия (например, «возле», «преимущественно» и пр.), т.к. это существенно упрощает процесс 
восприятия для человеческого языка и мышления;

- отсутствует потребность управления взаимной зависимости начальных параметров, на базовом уровне по большему счету определяющаяся субъективной точкой зрения специалиста.

Использование в практической деятельности аппарата интервальной математики [2] для описания неточностей, а также иных компонентов неточного программирования (не беря во внимание факт положительной скорости вычислительных возможностей) - все это В совокупности являет собой недостаточно точное средство; в основном это взаимосвязано с колебанием в интервале от 100\% к 0\% вероятности при пересечении интервальной границы. Используя вышеперечисленные принципы в практике решения различных задач разработки комплекса месторождений углеводородов в разных агрегатных состояниях, точным математическим комплексом, благодаря которому данную неточность можно делать формальной, является аппарат нечетких чисел.

\section{Цель исслеАования}

Целью исследования является анализ основных положений теории нечетких множеств, анализ применяемых элементов теории нечетких множеств при оценке прогнозирования запасов углеводородов, анализ возможности применения системной имитации движения категорийных запасов.

\section{Материал и методы исслеАования}

Ключевые аспекты и методы проектирования разработки месторождений углеводородов, представленные в трудах российских и зарубежных ученых, остаются фундаментальными по сей день. Абсолютно любой проект разработки газовых месторождений (РГМ) обязан охватывать вопросы объединения различных данных о залежи (сейсмические исследования, геофизические данные, лабораторная и промысловая информация); необходимы обоснование и отбор геологической базы - имитация залежи для макетирования; должны быть разграничены эксплуатационные объекты; выделены стадии перехода от естественных режимов к базисным способам воздействия; выбраны средства воздействия либо составные комплексы; выбрана сетка расстановки различных типов скважин; рассчитаны производственные и экономические составляющие альтернативных проектов и выбраны целесообразные варианты разработки. Наращивание положительного опыта выработки залежей в многообразии геологических условий, синтез новейших способов и методов воздействия создавали положительную среду для мо- дернизации знаний в моделировании разработки месторождений углеводородов, скурпулезной проработке некоторых аспектов проектирования.

Понятие «нечеткое множество». Нечёткое множество представляет собой понятие, употребленное впервые ученым Л. Заде в 1965 г. в материале «Fuzzy Sets» в издании «Information and Control», в котором Заде укрупнил базовое описание множества, предположив, что функция принадлежности элемента множеству свободно умещается в значениях интервала $[0,1]$. Нечеткое множеством $A$ представляет собой комплекс упорядоченных пар, которые скомпонованы из элементов $x$ универсального множества $X$ и гармонирующих степеней принадлежности $\mu_{A}(x)[1]$ :

$$
A=\left\{\left(x, \mu_{A}(x)\right) \mid x \in X\right\}
$$

более того, $\mu_{A}(x)$ - функция принадлежности (характеристическая функция), индицирующая, в какой мере (степени) конкретный элемент $x$ принадлежит множеству $A$ (нечеткому).

Предположим, что $A$ в данном случае - нечёткое множество с компонентами из универсального множества $X$ и множеством функциональных принадлежностей $M=[0,1]$, отсюда, суппорт (носитель) множества sирр $A$ - это множество

$$
\sigma(A)=\left\{x \mid x \in X, \mu_{A}(x)>0\right\} .
$$

$F$ - множествами общепринято называть комплекс полного ряда подмножеств $F(X)$ всякого множества $X$, отсюда вытекает - их функциями принадлежания являются $F$-функции. $C$ целью маркировки $F-$ множеств используют запись следующего вида: $A=\left\langle\mu_{A}(x), \sigma(A)\right\rangle$.

$$
\begin{gathered}
\text { Величина } \\
\text { sup } \mu_{A}(x) \\
x \in X
\end{gathered}
$$

является высотой нечёткого множества $\boldsymbol{A}$. Нечёткое множество $A$ нормально, при выполнении условия, что высота данного множества равна 1. В случае, когда высота строго менее 1, нечёткое множество субнормально.

Нечёткое множество пусто при выполнении условия, что:

$$
\forall_{x} \in X: \mu_{A}(x)=0
$$

Непустое субнормальное нечёткое множество допускается нормализовать формулой: 


$$
\mu_{A}^{\prime}(x)=\frac{\mu_{A}(x)}{\sup \mu_{A}(x)} .
$$

Нечёткое множество можно расценивать как унимодальное при том условии, если $\mu_{A}(x)=1$ (на одном $x$ из $X)$.

Элементы $\chi \in X$, для которых $\mu_{A}(x)=0.5$ - точки перехода множества $A$ (нечеткого).

В случае, когда $\mathrm{A} \in F$ и $\alpha \in[0,1], \alpha$-уровневым множеством $F$-множества $A$ является множество

$$
\omega_{\alpha}(A)=\left\{x \in X \mid \mathrm{M}_{A}(x) \geq \sigma\right\} .
$$

Вычислительные операции, в которых присутствуют нечеткие множества, являются своего рода обобщением для тех операций, которые осуществляются посредством базовых множеств. Все эти вычислительные операции зачастую дробятся на отдельные компоненты с численными значениями функции принадлежности. Наибольшей популярностью пользуются операции пересечения и объединения нечетких множеств, обусловленные такими операциями, как $\mathrm{min}$ и $\max$ над численными показателями принадлежности.

Пересечение нечётких множеств $A$ и $B$ представляет собой самое большое нечёткое подмножество, имеющее место одновременно в $A$ и $B$ :

$$
\mathrm{M}_{A \cap B}(x)=\min \left(\mathrm{M}_{A}(x), \mathrm{M}_{B}(x)\right) .
$$

Результат умножения нечётких множеств $A$ и $B$ является нечётким подмножеством, которое обладает функцией принадлежности:

$$
\mathrm{M}_{A B}(x)=\mathrm{M}_{A}(x) \mathrm{M}_{B}(x) .
$$

Объединение нечётких множеств $A$ и $B$-min приемлемое нечёткое подмножество, куда входят $A$ или $B$ :

$$
\mathrm{M}_{A \cup B}(x)=\max \left(\mathrm{M}_{A}(x), \mathrm{M}_{B}(x)\right) .
$$

Сумма нечётких множеств $A$ и $B$ представляет собой нечёткое подмножество, которое обладает функцией принадлежности:

$$
\mathrm{M}_{A+B}(x)=\mathrm{M}_{A}(x)+\mathrm{M}_{B}(x)-\mathrm{M}_{A}(x) \mathrm{M}_{B}(x) .
$$

Отрицание множества $A$ является аналогичным множеством $\bar{A}$, разнящееся тем, что оно включает в себя функцию принадлежности:

$$
\mathbf{M}_{\bar{A}}(x)=1-\mathrm{M}_{A}(x) \text { для каждого } x \in X .
$$

При выполнении операции дефазификации нечеткого множества нужной функции принадлежности $\mathrm{M}_{A}(x)$ сопоставляется конкретное детерминированное число, которое характеризует ее.

При практической реализации нечетких вычислений регулярно используемыми являются нечеткие треугольные числа, т.к. функция принадлежности, которой они обладают, по форме напоминает собой нечто, похожее на треугольник. В случае, когда специалист на гипотетическом уровне может указывать на то, что определенное недостоверное значение может умещаться в значениях $a 2-a 3$ (оно точно не будет меньше $a 1$ и больше $a 4$ ), записать данную гипотезу можно в виде треугольного нечеткого числа $A=\langle a 1, a 2, a 3, a 4\rangle$. В связи с чем треугольное нечеткое число $A=<a 1, a 2, a 3>$ будет представлять пример частного случая треугольного при $a 2=a 3$, интервальное [a1, a3] - при $a 1=a 2$, a3 = a4, а определенное значение - при $a 1=a 2=a 3=a 4$.

Уделим отдельное внимание неточному расширению области детерминированной задачи. Известный математик Лю [4] в своих исследованиях предложил неясное программирование, утверждая следующие виды программирования: нечеткое, неточное и стохастическое. Главная функция и лимитирование у многих видов неточного программирования определяются в виде четкого аналитического вида. При воспроизведении вычислительных операций, связанных с задачами максимизации выгодных характеристик суммарной добычи, предлагается переходить от розыска приемлемого решения (непрерывного случая) к поиску оптимального решения, в частности для дискретного случая. Ввиду отсутствия возможности указания точных данных ключевой функции и лимитирования, придется исключить использование способов неточного варианта программирования. Принимая данные сведения в качестве актуальных, предлагается сохранить базовую схему, диалоговый интерфейс, информационное и программное обеспечения, осуществленные для конкретного варианта, где произведена замена конкретных числовых значений и операции над ними соответствующими нечеткими числами и нечеткой математикой $[3,4]$. Важно учитывать такие аспекты, как:

- не все начальные значения следует рассматривать с точки зрения нечеткости;

- максимизирующие и имитационные средства должны иметь тождественный диалоговый интерфейс, информационное и программное обеспечение.

Петрофизические нечеткие модели расписывают экспериментальные данные в форме функций принадлежности для нечеткого детерминирования между различными 
геолого-физическими параметрами. Этот тип моделей не является традиционным, и при их построении возникает необходимость в применении большого массива данных. Однако, несмотря на эти недостатки, петрофизические нечеткие модели несут в себе достаточно высокую степень наглядности, а также дают возможность получать дифференцированный (по степени достоверности) для каждого прогнозного параметра конечный результат. Исходя из изложенного, эти модели дают возможность выполнения контроля за обеспеченностью исходными параметрами и получения оценки реальности полученных геологических имитационных моделей. Это дает достижимость многовариантных построений геолого-технических моделей, отражающих достоверную информацию.

Нечеткие множества как способ представления категорий аккумуляции природного газа.

Вопрос, касающийся точности оценки запасов, до сих пор актуален. Рационально оценить количество необходимых ресурсов природного газа, представленного в виде различных категорий - вот главная проблема в области максимизации разработки комплекса месторождений природного газа.

Системная модель передвижения запасов зависит от категорий. Перед тем, как начать работать с какой-либо категорией, следует уразуметь тот факт, что любая категория является носителем информации, которая дает характеристику определенному уровню знаний запасов природного газа, размещенного в определенном отложении каких-либо природных ископаемых. Рассматривая это с позиции системных или информационных гипотез, становится очевидным факт того, что неточность каких-либо данных точно описывает энтропию. Энтропия ресурсных комплексов и запасов на перманентной базе в процессе движения начинает постепенно уменьшаться от самой низкой категории ресурсов D2 (опираясь на Классификацию [3]) к самой высокой категории запасов A.

Проведем небольшую мыслительную операцию: разделим имеющееся множество объектов добычи газа М на 9 подмножеств $\{K j\}, j=0,1,2, \ldots, 8$, которые между собой являются параллельными. Элементы всех подмножеств - аккумулированные объекты с запасами конкретной категории, объем которых показан в виде нечетких множеств. Категория $D 2$ соответствует подмно- жеству $K 1$, подмножеству $K 2-D 1, K 3-C 3, K 4-C 2$, $K 5-C 1, K 6-B, K 7-A$. В комплекс $\{K j\}$ необходимо включить подмножество $K 0$ (объекты с отсутствием информации о величине запасов), и подмножество $K 8$ (объекты с полностью извлеченными запасами).

Учитывая, что в ходе разработки вспомогательные блоки информации о запасах в объектах выделяется посредством накопительного эффекта, то можно заключить, что это обусловливает перманентные модификации в разделении множества М. Для более полного представления важно рассматривать данные подмножества $\{K j\}$ в разные временные промежутки $t$ как изменяющиеся подмножества $\{K j(t)\}$, которые имеет следующие характеристики:

- если $a \in K j(\phi)$, где $j=1,2, \ldots, 8$, то при любых $t>\mathrm{p}, i<j$ объект $a \notin K j(t)$;

- если $a \in K j(\phi)$, где $j=1,2, \ldots, 7$, то существует $t>$ ф такое, что объект $a \epsilon K j+1(t)$.

Не следует забывать: чтобы оценить извлекаемые запасы природного газа, зачастую используют объемный способ, также существует его истолкование, гарантирующий учет неточности исходных данных на базе использования нечетких множеств [6].

\section{Результаты исслеАования \\ и их обсужАение}

Принимая результаты вышеперечисленных исследований в качестве актуальных, применение теории нечетких множеств для оценки геолого-технологических компонентов в ситуации наличия в заданных уравнениях неточно введенных величин предоставляет допустимость получить количественную оценку неопределенности оцениваемых факторов и создает основу для принятия четко обоснованных решений при наличии у эксперта выбора из нескольких моделей.

\section{Эак^ючение}

Совокупность нечетких множеств органично встроится в процесс организации тактик разработки комплекса нефтяных и газовых месторождений при условии учета неточности начальных данных, в том числе ресурсы газа широкой категорийности. Для этого следует применять нечеткое расширение области определенных задач, касающихся моделирования.

\section{ЛИТЕРАТУРА}

1. Алтунин А.Е., Семухин М.В. Модели и алгоритмы принятия решений в нечетких условиях: Монография. Тюмень: Издательство Тюменского государственного университета, 2015.- 352 с.

2. Желтов Ю.В. и др. Разработка и эксплуатация нефтегазоконденсатных месторождений. Москва: Недра, 2013.— 254 с. 
3. Скиба А.К., Соломатин А.Н. Моделирование и оптимизация стратегий разработки группы газовых месторождений. М.: ВЦРАН, 2012.- 40 с.

4. Лю Б. Теория и практика неопределенного программирования. М.: БИНОМ, 2013. -416 с.

5. Кофман А. Введение в теорию нечетких множеств. Пер. с франц. - Москва: Радио и связь, 1982. - 432 с.

6. Хачатуров, В.Р. Планирование разработки группы газовых месторождений с учетом неопределенности исходных данных [Электронный ресурс] // Экспозиция Нефть Газ: электрон. науч. журн. 2015. № 2 (41). С. 20-23. URL: http://runeft.ru (дата обращения: 19.05.2021)

( ) Чувашов Артур Александрович ( sid_artur@mail.ru ), Кожевникова Полина Валерьевна ( pkozhevnikova@ugtu.net ). Журнал «Современная наука: актуальные проблемы теории и практики»

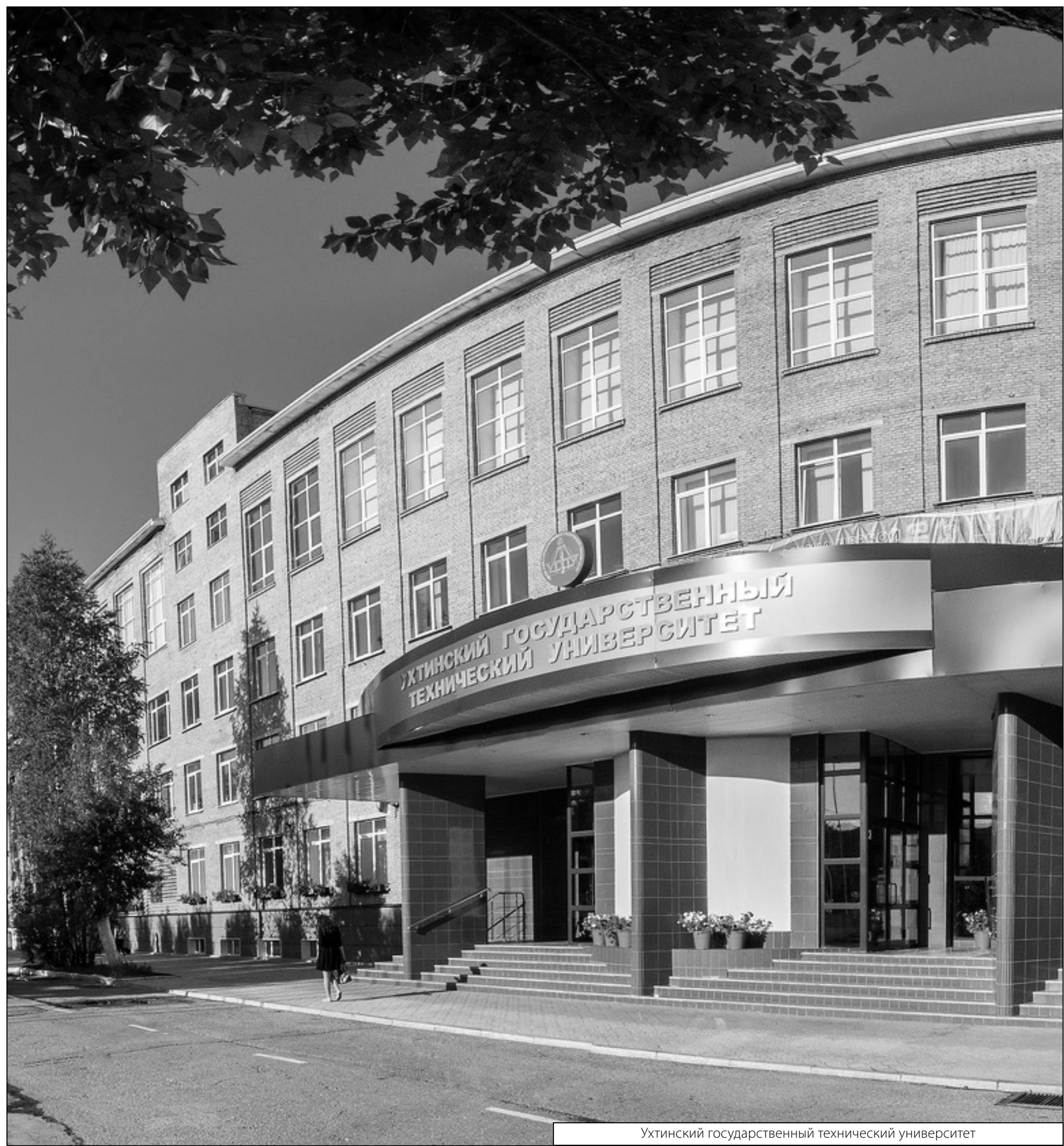

